\title{
Squamous cell carcinoma of the suprapubic tract: A rare presentation in patients with chronic indwelling urinary catheters
}

\author{
Peter Alexander Massaro, MD, MASc, Jonathan Moore, MD; ${ }^{*}$ Tarek Rahmeh, MD; \\ Michael J. Morse, MD, FRCSC
}

*Department of Urology, Dalhousie University, Halifax, NS; §Department of Pathology, Dalhousie University, Halifax, NS; and Department of Laboratory Medicine, Saint John Regional Hospital, Saint John, NB; * Department of Urology, Dalhousie University, Halifax, NS and Saint John Regional Hospital, Saint John, NB

Cite as: Can Urol Assoc J 2014;8(7-8):e510-4. http://dx.doi.org/10.5489/cuaj.1637

Published online July 18, 2014.

\section{Abstract}

Squamous cell carcinoma (SCC) of the bladder is uncommon, but can arise in the setting of long-term bladder catheterization and chronic inflammation. SCC can arise primarily from the suprapubic catheter tract, but fewer than 10 such cases have been reported. We document 2 cases of SCC arising from the suprapubic tract associated with chronic indwelling urinary catheters. SCC must be differentiated from granulomatous conditions, which are quite common in patients with suprapubic catheters.

\section{Introduction}

Squamous cell carcinoma (SCC) of the bladder is a rare condition in the general population, making up about $5 \%$ of all bladder tumours. ${ }^{1}$ Risk factors for SCC of the bladder include states of chronic inflammation and infection, such as long-term indwelling catheters or infection with Schistosoma haematobium primarily in endemic regions. ${ }^{2}$ A higher incidence of SCC in the spinal cord injury (SCI) population has been documented on the order of $2 \%$ to $10 \%$ beyond 10 years of catheterization. ${ }^{3-6}$ Among patients with $\mathrm{SCl}$, about $50 \%$ of cases of primary bladder cancer are SCC. ${ }^{5}$ The risk is thought to stem from irritation and inflammation related to the chronic use of both urethral and suprapubic catheters ${ }^{3,7}$ chronic infections, ${ }^{8}$ bladder stones ${ }^{8}$ or, possibly, the inherent pathology of the neurogenic bladder itself. ${ }^{5}$

Although patients requiring long-term indwelling catheters are at increased risk of developing SCC, those with suprapubic catheters may more rarely develop SCC arising primarily from the suprapubic tract itself. This has been described in only a handful of cases. ${ }^{9-13}$ We present 2 additional patients who developed SCC arising from their supra- pubic catheter sites and discuss their surgical management and outcomes.

\section{Case 1}

A 55-year-old male, with a history of paraplegia secondary to spinal tuberculosis acquired at 16 years of age, presented with a 15-month history of an expanding, large, fungating lesion encircling his suprapubic catheter site. The catheter had been inserted within the first year of the onset of paraplegia. The patient's history was otherwise unremarkable and he was a lifelong non-smoker. There was no history of travel to areas endemic with Schistosoma haematobium. Examination of the lesion revealed an area of $15-\mathrm{cm}$ in maximum diameter around the catheter site (Fig. 1). A biopsy of the lesion was diagnostic for SCC.

Cystoscopy revealed a contracted bladder, but no evidence of stones, fistulae, or tumours of the mucosa or suprapubic tract. Biopsies of the bladder and suprapubic tract were negative for malignancy. Computed tomography (CT) showed infiltration of the mass into the anterior bladder wall, extending into the deep subcutaneous tissue and abdominal wall musculature (Fig. 2). Lymph nodes were negative. Subsequent bone scan was negative for bony metastases.

Excision of the tumour was carried out by both the urology and plastic surgery services. Intra-operatively, the tumour invaded the anterior rectus fascia, rectus abdominus, periosteum of the pubic symphysis and spermatic cords bilaterally. Induration of the anterior and posterior surfaces of the bladder was suggestive of tumour involvement. Given the extent of disease, complete excision of the tumour was not deemed possible. A conservative surgical approach was followed, consisting of resection of the grossly palpable tumour of the extra-vesicle bladder wall. Postoperatively, the patient developed a vesicocutaneous fistula, delaying the administration of adjuvant radiotherapy which he subsequently declined. 


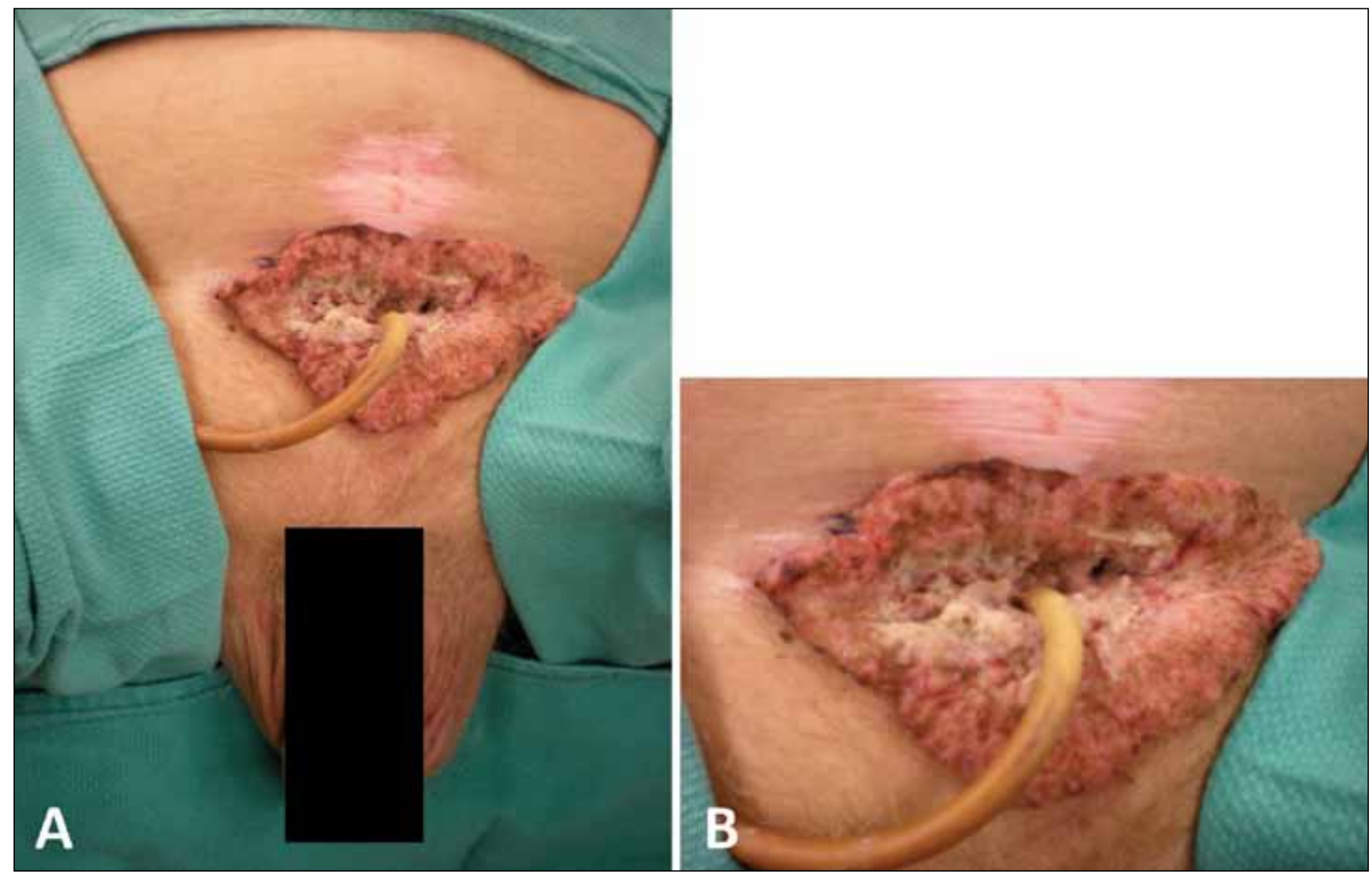

Fig. 1. A: Squamous cell carcinoma of the suprapubic tract in a 55-year-old male paraplegic patient; B: Close-up image of the fungating lesion.

Surgical pathology confirmed the diagnosis of an invasive SCC with clear surgical margins (Fig. 3); however within a year post-resection, the patient presented with local tumour recurrence, bilateral ureteric obstruction with hydroureteronephrosis and renal insufficiency. Nephrostomy tubes were inserted bilaterally. No further treatment was administered and a palliative approach was pursued.

\section{Case 2}

An 85-year-old female presented with a complaint of increased discharge around her suprapubic catheter site. The catheter had been inserted 9 months prior for idiopathic urinary retention. Her medical history was significant for Type II diabetes and she was a smoker. There was no history of Schistosoma haematobium exposure. On presentation, there was a $4-\mathrm{cm}$, fungating tumour surrounding the catheter site (Fig. 4, part A). Superficial biopsies were obtained and were diagnostic for SCC. Cystoscopy revealed no evidence of tumour.

Excision of the suprapubic mass was carried out initially under local anesthetic. Surgical margins were subsequently positive and therefore a wider local excision was performed under a general anesthetic with a partial cystectomy because of tumour extension into the bladder dome (Fig. 4, part B). Pathology revealed invasive SCC with deep surgical margins remaining positive (Fig. 5). Postoperatively, the patient experienced a protracted hospital course complicated by the development of congestive heart failure, a myocardial infarction and acute kidney injury, requiring management in the intensive care unit. A subsequent CT scan revealed lytic lesions of the pubic symphysis consistent with local tumour invasion. Eventually recovering from her postoperative course, the patient requested no further treatment, and elected for a palliative approach.

\section{Discussion}

SCC of the bladder is a rare disease in the general population, accounting for only $5 \%$ of all bladder primary tumours. ${ }^{1}$ In the setting of chronic indwelling catheters in the SCI population, it accounts for 10 times more cases of bladder cancer, with an estimated incidence of $2 \%$ to $10 \%$. $^{3-6}$

Rarely, in the setting of chronic suprapubic catheterization, SCC may arise primarily from the suprapubic cystotomy tract. Similar to primary SCC of the bladder, SCC of the 


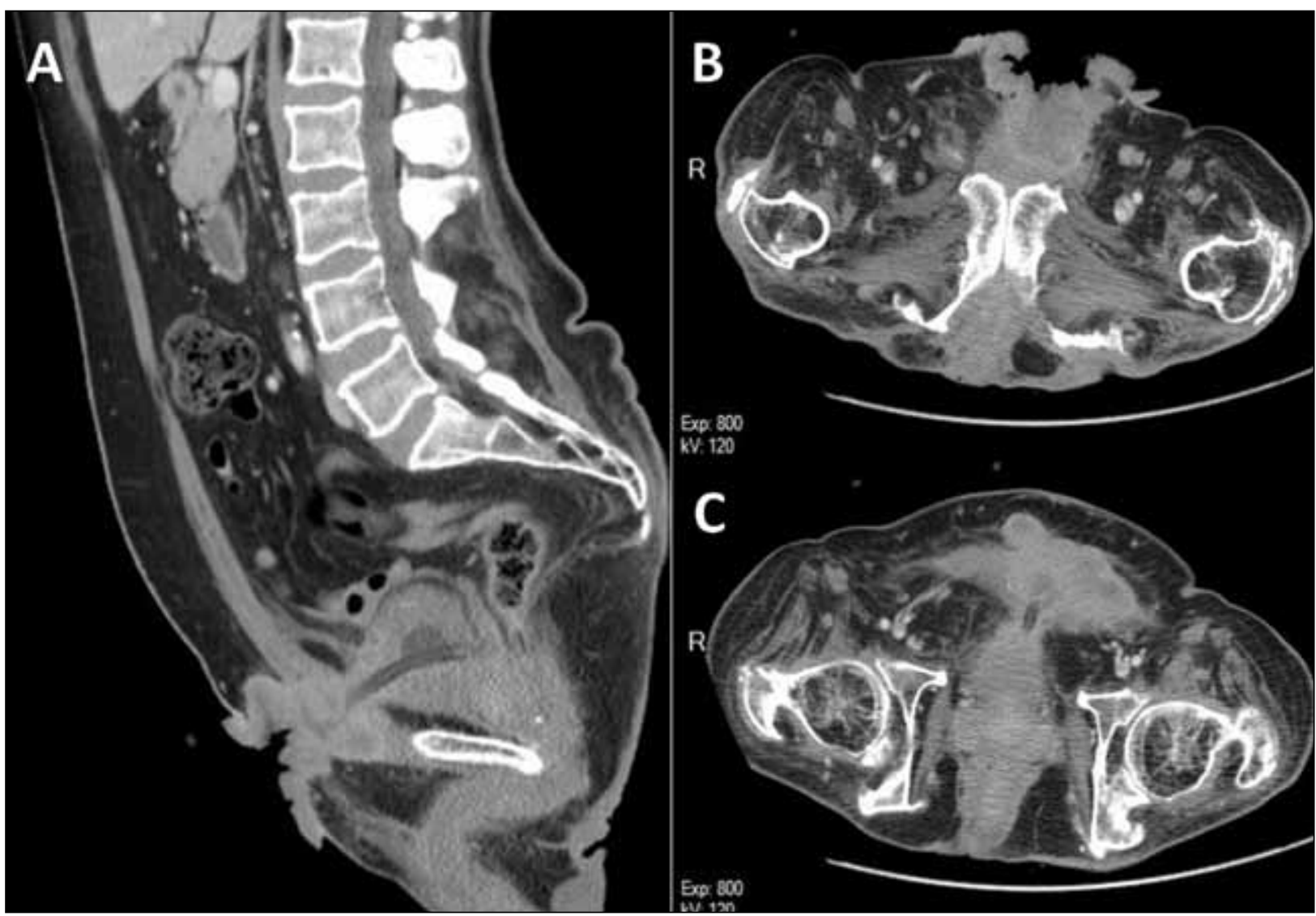

Fig. 2. A computed tomography scan of a 55-year-old paraplegic patient with squamous cell carcinoma the suprapubic tract. A: Sagittal section demonstrating fungating lesion invading the rectus abdominus with extension to the anterior border of the pubic bone and dome of bladder. The suprapubic catheter and balloon can be observed. B-C: Transverse sections further delineating the extent of the tumour.

suprapubic tract likely arises from chronic irritation and inflammation of the tract and surrounding skin. ${ }^{7}$ This was first described by Stoumbakis and colleagues in $1993 .{ }^{9}$ They presented a patient who required suprapubic catheterization for urethral strictures. Five years after the insertion, SCC was found at the cystotomy site with extension into the

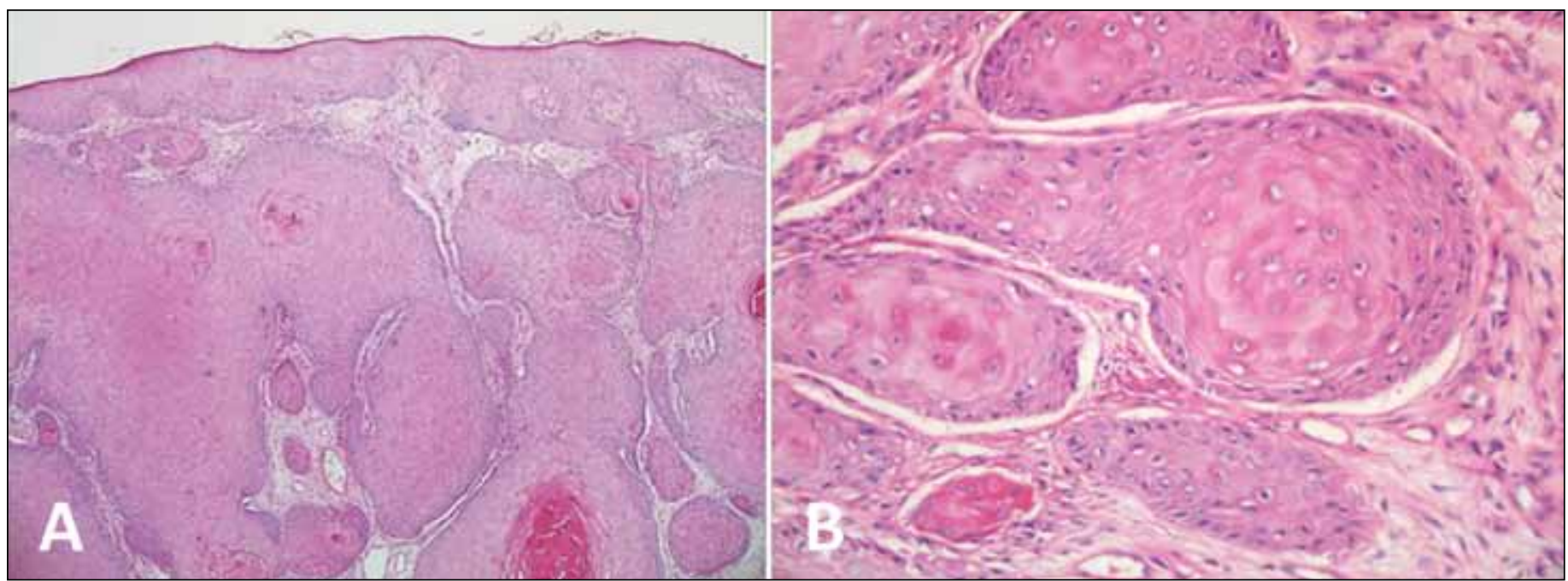

Fig. 3. This well-differentiated tumour forms large irregularly shaped islands pushing deep into the underlying soft tissue (A). A high-power microscopic view shows that nuclear pleomorphism is minimal, mitoses are rare, and keratinization is evident (B). 


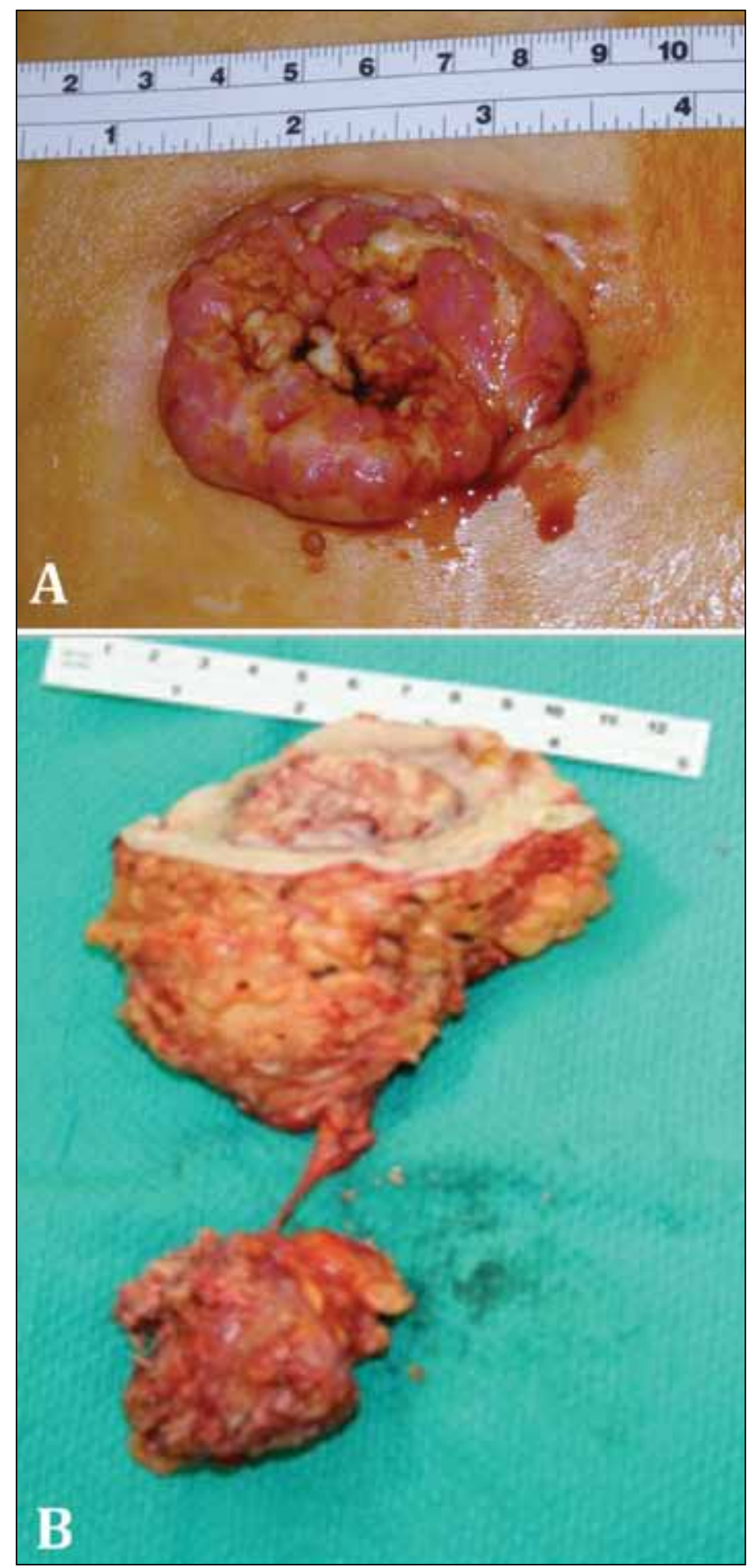

Fig. 4. Squamous cell carcinoma arising from the suprapubic catheter site in an 85 -year-old female only 9 months after catheter placement. A: Appearance of lesion on initial presentation. B: Postoperative specimen including the skin lesion and subcutaneous tissues (top), suprapubic tract (middle), and partial cystectomy specimen (bottom).

abdominal wall, but no bladder involvement. Subsequent reports of isolated cases from 1995 to 2011 describe cases of SCC arising from the suprapubic tract in patients with
SCls and suprapubic catheters. ${ }^{10-13}$ The average time from catheter placement to presentation was 31 years, which is in concordance with the typical time of presentation of SCC in the chronic indwelling catheter population. ${ }^{3,5,6}$ Only 1 case demonstrated evidence of intravesical disease, ${ }^{13}$ while another demonstrated extension, but not penetration of SCC into the detrusor muscle. ${ }^{12}$ Four cases were treated with wide excision $^{9,10,12,13}$ and either partial ${ }^{9,10}$ or radical ${ }^{12,13}$ cystectomy, with or without neoadjuvant radiotherapy. Palliative radiotherapy was provided to the remaining patient who had positive nodal and metastatic disease on CT. ${ }^{11}$

In our series, we presented 2 patients with SCC arising from their suprapubic cystotomy tracts. Both patients underwent partial cystectomy, but demonstrated subsequent disease persistence. Both declined radiotherapy and chose a palliative approach to their care. The male patient developed SCC about 39 years after suprapubic catheter placement. The late presentation of his disease is in keeping with observed trends of bladder cancer development in the SCI population..$^{3,5,6}$ In contrast, the female patient in this series developed SCC at the suprapubic tract only 9 months after catheter placement. In the latter case, it is difficult to explain such an early presentation of SCC. Nevertheless, it should be recognized that the duration of catheterization increases the risk of bladder cancer, not only intravesically, but also along the suprapubic tract. It would be prudent for patients, caregivers and healthcare practitioners to maintain a high index of suspicion if an irregular, expanding, or otherwise suspicious lesion is identified at the suprapubic site regardless of the duration of catheterization. Such lesions must be differentiated from granulomatous changes seen commonly at the suprapubic site.

Surgical resection-radical cystectomy, or, in appropriate candidates, partial cystectomy-is the recommended management of SCC of the bladder with or without adjuvant radiotherapy. ${ }^{14}$ Other therapies, such as neoadjuvant radiotherapy, either lack sufficient evidence to support their routine application, or, in the case of chemotherapy, do not provide a demonstrated disease response. ${ }^{14}$ Due to the sporadic occurrence of primary SCC of the suprapubic tract, we can only extrapolate a similar treatment approach in this setting. The 2 patients in this series underwent wide local excision of the surrounding skin and suprapubic tract and partial cystectomy. They were offered, but declined, adjuvant radiotherapy.

Although there is a lack of consensus regarding optimal urological follow-up of $\mathrm{SCl}$ patients with indwelling suprapubic catheters, some have recommended annual cystoscopic surveillance with biopsies of suspicious intra-vesical lesions. ${ }^{7}$ SCC of the suprapubic tract is likely under-reported, particularly when it occurs in conjunction with already identified intravesical disease. Therefore, regular assessment of the suprapubic catheter site at the time of scheduled followup and/or catheter change should be carried out. 


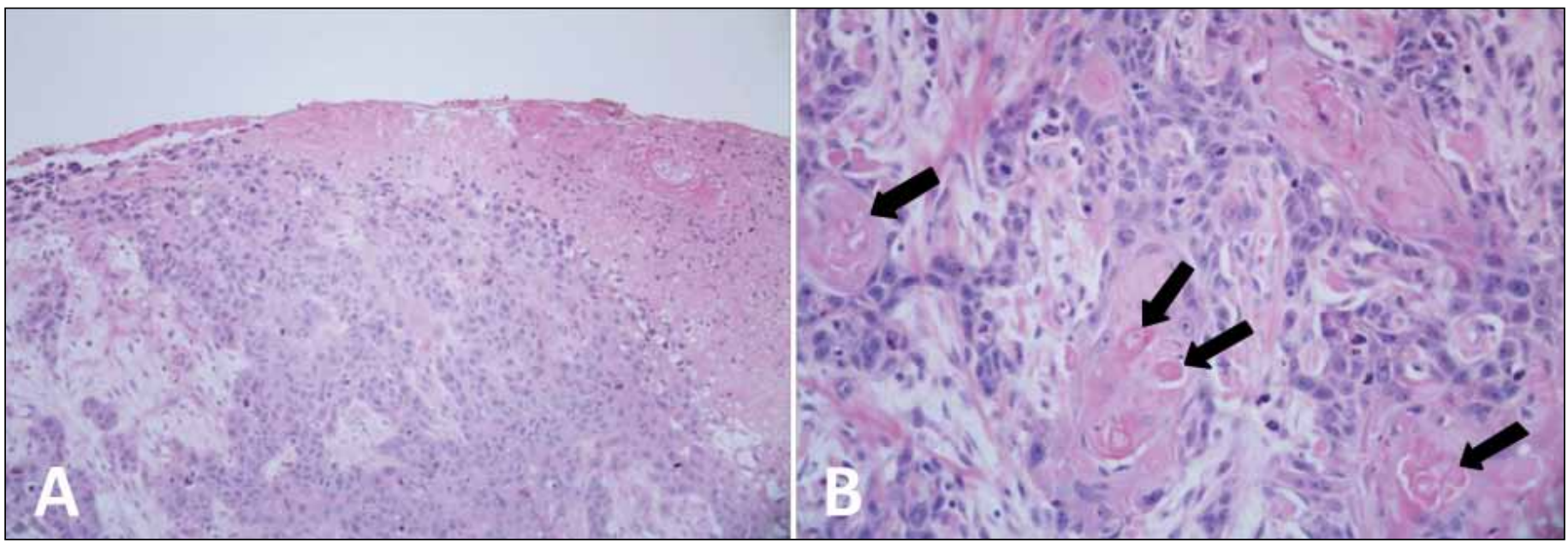

Fig. 5. Ulceration in the suprapubic tract with extensive involvement by infiltrating carcinoma. Necrosis is noted on the right (A). The tumour forms jagged sheets of pleomorphic cells and mitoses are frequent. Despite the aggressive cytological features, the presence of keratin pearls (arrows) is a clear evidence of squamous differentiation (B).

\section{Conclusion}

SCC of the suprapubic tract is a rare malignant presentation in patients with chronic suprapubic catheters. In addition to the 5 cases reported worldwide, we have added 2 additional cases of SCC of the suprapubic tract to the medical literature. Extrapolating from the treatment of primary SCC of the bladder, surgery and radiotherapy are the recommended treatment modalities for SCC of the suprapubic tract. A high index of suspicion for abnormal lesions arising from the suprapubic catheter site and differentiation from common granulomatous changes at the time of follow-up or catheter change would lead to earlier identification and treatment of the disease process.

Competing interests: Dr. Massaro, Dr. Moore, Dr. Rahmeh and Dr. Morse all declare no competing financial or personal interests.

This paper has been peer-reviewed.

\section{References}

1. Rous SN. Squamous cell carcinoma of the bladder. J Urol 1978;120:561-2.

2. Abol-Enein H. Infection: Is it a cause of bladder cancer? Scand J Urol Nephrol Suppl 2008;42:79-84. http://dx.doi.org/10.1080/03008880802325309

3. Kaufman JM, Fam B, Jacobs SC, et al. Bladder cancer and squamous metaplasia in spinal cord injury patients. J Urol 1977;118:967-71.
4. Bejany DE, Lockhart JL, Rhamy RK. Malignant vesical tumors following spinal cord injury. J Urol 1987;138:1390-2.

5. Kalisvaart JF, Katsumi HK, Ronningen LD, et al. Bladder cancer in spinal cord injury patients. Spinal Cord 2010;48:257-61. http://dx.doi.org/10.1038/sc.2009.118

6. Locke JR, Hill DE, Walzer Y. Incidence of squamous cell carcinoma in patients with long-term catheter drainage. J Urol 1985; 133:1034-5.

7. Feifer A, Corcos J. Contemporary role of suprapubic cystostomy in treatment of neuropathic bladder dysfunction in spinal cord injured patients. Neurourol Urodyn 2008;27:475-9. http://dx.doi.org/10.1002/ nau.20569

8. Groah SL, Weitzenkamp DA, Lammertse DP, et al. Excess risk of bladder cancer in spinal cord injury: Evidence for an association between indwelling catheter use and bladder cancer. Arch Phys Med Rehabil 2002;83:346-51. http://dx.doi.org/10.1053/apmr.2002.29653

9. Stroumbakis N, Choudhury MS, Hernandez-Graulau JM. Squamous cell carcinoma arising from suprapubic cystotomy site without bladder involvement. Urology 1993;41:568-70. http://dx.doi. org/10.1016/0090-4295(93)90107-L

10. Schaafsma RJ, Delaere KP, Theunissen PH. Squamous cell carcinoma of suprapubic cystostomy tract without bladder involvement. Spinal Cord 1999;37:373-4. http://dx.doi.org/10.1038/si.sc.3100834

11. Ito $\mathrm{H}$, Arao $\mathrm{M}$, Ishigaki $\mathrm{H}$, et al. A case of squamous cell carcinoma arising from a suprapubic cystostomy tract. BMC Urol 2011;11:20. http://dx.doi.org/10.1186/1471-2490-11-20

12. Stokes $S 3 r d$, Wheeler JS Jr, Reyes CV. Squamous cell carcinoma arising from a suprapubic cystostomy tract with extension into the bladder. J Urol 1995;154:1132-3. http://dx.doi.org/10.1016/S0022$5347(01) 66999-4$

13. Auffenberg GB, Meeks JJ, Smith ND. Suprapubic mass in patient with neurogenic bladder due to spinal cord injury managed with indwelling suprapubic tube. Urology 2009;73:457-60. http://dx.doi. org/10.1016/j.urology.2008.10.054

14. El-Sebaie M, Zaghloul MS, Howard G, et al. Squamous cell carcinoma of the bilharzial and non-bilharzial urinary bladder: A review of etiological features, natural history, and management. Int I Clin Oncol 2005;10:20-5. http://dx.doi.org/10.1007/s10147-004-0457-6

Correspondence: Dr. Peter Alexander Massaro, Department of Urology, Dalhousie University, Halifax, NS; massaro.peter@gmail.com 